\title{
LA EDUCACIÓN, ENTRE LA CIENCIA Y LA TÉCNICA
}

\author{
José Alberto Rivera Piragauta ${ }^{1}$ \\ Universidad Nacional Abierta y a Distancia - Colombia \\ josea.rivera@unad.edu.co
}

Recepción: 05/09/2012

Evaluación: 29/09/2012

Aceptación: 15/11/2012

Artículo de Revisión

doi: http://dx.doi.org/10.9757/Rhela.19.07

\section{RESUMEN}

Este artículo plantea una reflexión filosófica sobre la educación. Pretende ser un acercamiento, a modo de disertación, redescubriendo el papel que tiene la educación cuando se mueve en coordenadas tan claramente definidas como lo es la ciencia y la técnica, sin desconocer que la mirada está puesta en el horizonte de la educación desde la virtualidad. La metodología consistió en la revisión y el posterior análisis de algunos textos que se referencian a lo largo del escrito, y que son posibles caminos que orientan al lector en temas interesantes y a su vez actuales sobre la educación. Así las cosas, la hipótesis a desarrollar es: El objeto técnico es el punto de encuentro de la ciencia y la técnica que justifica la nueva metodología de la educación en los ambientes

\section{EDUCATION, BETWEEN SCIENCE AND TECHNIQUE}

\section{ABSTRACT}

This paper establishes a philosophical analysis towards education. It also pretends to be an approach even a disclosure by rediscovering the role of education while following specific coordinates such as science and te- virtuales de aprendizaje. Es una reflexión que se fundamenta en la riqueza propia de la historia del pensamiento humano y de sus aportes a la ciencia y, por ende, al conocimiento, si y solo si tiene su necesaria réplica en la educación. Dicha reflexión tiene una carga bastante fuerte en literatura filosófica, que sirve de base para elaborar un posterior discurso sobre la educación en medio del mundo tecnológico, ya familiar para todo el género humano. Basta, por ahora invitar al lector a descubrir las aristas y los puntos de interés para propiciar nuevas indagaciones sobre el tema.

Palabras claves: Revista Historia de la Educación Latinoamericana, ciencia, técnica, educación, filosofía.

\section{A EDUCAÇÃO, ENTRE A CIÊNCIA E A TÉCNICA}

\section{RESUMO}

Este artigo apresenta uma reflexão filosófica sobre a educação. Pretende ser uma aproximação, à guisa de dissertação, redescobrindo o papel que tem a educação quando se move em coordenadas tão claramente

\footnotetext{
Magíster en Filosofía de la Pontificia Universidad Javeriana. Actualmente vinculado como docente a la Universidad Nacional Abierta y a Distancia - UNAD en la Escuela de Ciencias de la Educación en los programas de posgrado en Educación y de la Licenciatura en Filosofía. Carrera $14 \mathrm{~N}^{\circ} 14 S-23$ Bogotá - Colombia. Miembro del grupo de investigación UBUNTU avalado por Colciencias, cuyo interés de investigación es la educación y la tecnología. Ha escrito algunos artículos en la línea de pedagogías mediadas sobre todo en el estudio de la influencia de la tecnología en la educación.
} 
chnique, not forgetting the main focus on virtual education. Methodology was constituted of review and analysis of some of the texts quoted in this article, as potential ways to lead readers into interesting and trend topics on education. As topics are established, hypothesis in development would be: Technical object as meeting spot for science and technique justifying new education technology in online learning environments.

As a fundamental consideration based on history richness of mankind thought and its endorsement to science and so to knowledge, only possible if it has its own replica in education field.

Such reflection has a heavy load in philosophical literature, which may help as a foundation for elaborating a new lecture on education in today's technological world, already familiar for all humans.

Last, for now our invitation to the reader in order to discover all edges and main ideas in order to give new approaches on the subject.

Key words: History of Latin American Education Journal, Science, technique, education, philosophy. definidas como o é a ciência e a técnica, sem desconhecer que o olhar está posto no horizonte da educação a partir da virtualidade. A metodologia consistiu na revisão e posterior análise de alguns textos que referenciam ao longo do texto, e que são caminhos possíveis que orientam o leitor em temas interessantes, e por sua vez atuais sobre a educação. Desta forma, a hipótese a desenvolver é: o objeto técnico é o ponto e encontro da ciência e da técnica que justifica a nova metodologia da educação nos ambientes virtuais de aprendizagem. É uma reflexão que se fundamenta na riqueza própria da historia do pensamento humano e de suas contribuições à ciência e, por conseguinte, ao conhecimento, se existe somente sua necessária réplica da educação. Esta reflexão tem uma carga bastante forte na literatura filosófica, que serve de base para elaborar um posterior discurso sobre a educação em meio ao mundo tecnológico, já familiar para todo o gênero humano. Basta, por hora, convidar o leitor a descobrir as arestas e os pontos de interesse para propiciar novas pesquisas sobre o tema.

Palavras-chave: Revista História da Educação Latino-americana, ciência, técnica, educaşão, filosofia.

\section{INTRODUCCIÓN}

El recurrente mito prometeico en los temas en que se discurre sobre las capacidades humanas, vuelve a tener eco. Cuenta la mitología griega ${ }^{2}$ que Prometeo le había robado el fuego a Hefestos y que luego se lo dio a los hombres junto con la sabiduría de Atenea, regalo que posteriormente desembocaría en el misterioso final de la caja de Pandora. Fue así como ellos tuvieron lo necesario para inventar y construir artefactos y hacer más habitable su mundo. La ciencia y el saber hacer de la técnica tienen en el mito prometeico la justificación fundante del pensamiento humano que crea. ¡Qué gran fortuna, entonces, significó este robo!

Son tres las partes que articulan este escrito: en primer lugar se presenta una manera de entender la relación entre epistemología y tecnología,

2 Juan Humbert, Mitología griega y romana (Barcelona, Editorial Gustavo Gili, S. A., 1997), 115. 
que se titula uniendo los términos. En segundo lugar se hace una reflexión filosófica sobre la tecnología, teniendo como centro el objeto técnico; por último, se ubica la educación entre la ciencia y la técnica, y se le denomina tecnología educativa.

\section{Uniendo los términos}

La educación, entre la ciencia y la técnica es el título que convoca la reflexión en este escrito. Son tres los términos que se relacionan y que a primera vista parecieran guardar cierta sinonimia, para lo que se hace necesario clarificar lo siguiente: No son auto-implicativos y por tanto no son equivalentes, no implica una gradación de importancia de la epistemología sobre la tecnología ${ }^{3}$ o de estas sobre la enseñanza; tampoco señalan una necesidad sine qua non, porque son términos con una etimología y una historia independiente.

La epistemología puede tener dos modos de ser comprendida y estudiada, uno, el que propone Mario Bunge en su libro Epistemología y otro el que proponen Mardones y Ursúa en su libro Filosofía de las ciencias humanas y sociales, haciendo la salvedad de que no son los únicos modos. Para la cuestión que aquí interesa se convierten en directrices válidas, sobre todo porque los tres autores abordan el tema de lo tecnológico desde la epistemología.

El prefacio de Bunge da a la ciencia el carácter de eje de la cultura contemporánea y motor de la tecnología. La epistemología es definida como ciencia de las ciencias o filosofía de la ciencia -sentido en el que se entenderá a lo largo de este escrito- ha gozado en los últimos años del rigor académico de diferentes facultades de filosofía y del de otros saberes. Para Bunge la filosofía de la ciencia «es la rama de la filosofía que estudia la investigación científica y su producto, el conocimiento científico» ${ }^{4}$. Bunge ubica la génesis de una reflexión epistemológica sería en el Círculo de Viena (el Wiener Kreis de 1927), que trataba de enfatizar en el empirismo lógico, y por tanto, en el lenguaje como factor

\footnotetext{
Tecnología se entiende en este escrito como el discurrir sobre la técnica, más adelante se desarrolla esta acepción del término.

4 Mario Bunge, Epistemología, 15. 
determinante en los procesos científicos. A este círculo pertenecieron nombres como M. Schlick, R. Carnap, H. Reinchenbach, V. Kraft ${ }^{5}$ y posteriormente con otros matices estará K. Popper. Pero, ¿cuál fue el aporte del círculo de Viena? Fue precisamente continuar un poco con la línea galileana de experimentar y de demostrar, como también lo había pregonado B. Russell tecnificando el lenguaje de la filosofía, buscando algo así como la cuadratura del lenguaje científico. Empero, este marcado interés fue al mismo tiempo la causa de su rápido declive pues la «filosofía lingüística mató al Círculo de Viena desde adentro» ${ }^{6}$ creando una brecha entre filósofos y científicos.

¿Qué importancia tiene actualmente la ciencia de las ciencias? Para Bunge, el epistemólogo asume la responsabilidad de dinamizar la ciencia y la reflexión en torno a esta. Permite la cercanía entre la filosofía y la ciencia generando criterios y puntos de encuentro en donde interviene la filosofía en los distintos campos del saber para robustecer y validar las propuestas científicas. La epistemología permite dar un rigor al discurso filosófico haciendo que sus verdades sean coherentes con la realidad de la que se admira y sobre la cual se indaga.

Por su parte, Mardones y Ursúa ${ }^{7}$ plantean el asunto desde la tradición aristotélica, con un corpus sistémico ya elaborado; sin embargo, se dirá que hay epistemología desde el momento mismo en que el hombre fue capaz de calcular las modificaciones que podía hacer al entorno que habitaba. Es la característica del homo faber, o del hombre primitivo, que como animal creador de herramientas transformaba con estas el ambiente primigenio. En necesaria relación con el homo faber estará el homo sapiens, lo que implica que no se trata solo de crear sino de saber crear y saber hacer, concepto que acuña y explica muy bien Aristóteles en la tarea del artesano. Con Aristóteles se sabe que se dio un giro a la reflexión filosófica en torno al conocer, pues lo real es fuente de conocimiento y la experiencia cobra un protagonismo fundamental y la

Bunge, Epistemología, 15.

Bunge, Epistemología, 17.

Estos autores comprenden la Epistemología no como un sistema dogmático a priori que determine lo que es el conocimiento, es de carácter dinámico y holístico. José M. Mardones y Nicolás Ursúa,Filosofía de las ciencias humanas y sociales(Barcelona: Anthropos, 1983), 67. 
realidad es susceptible de ser conocida según el modo de abordarla. Es así como el saber hacer (tekne) del artesano, encuentra en la filosofía aristotélica la palanca arquimédica para desarrollar el conocimiento. El artista crea (poiesis), produce cosas o arte-factos, modifica la realidad. La ganancia que se tiene con Aristóteles está en la posibilidad de la experiencia sensorial para poder generar aprendizaje y conocimiento. Pero, «la experiencia, al parecer, se asimila casi a la ciencia y al arte. Por la experiencia progresan la ciencia y el arte en el hombre. La experiencia, dice Polus, y con razón, hizo el arte, y la inexperiencia el azar» ${ }^{8}$. La experiencia permite que el arte se pueda enseñar o transmitir, y es entonces comunicable pues el maestro -que sabe hacer- puede mostrar el objeto creado y los discípulos saben que lo conocido se puede hacer. Entonces, el arte de saber hacer es para Aristóteles Ciencia o Episteme.

Ahora bien, es importante que esta ciencia posea un elemento sistémico que la configure y facilite su comprensión. Por lo tanto,

No babrá que buscar tanto la sustancia subyacente a los fenómenos cuanto las leyes matemáticas que nos desvelen la estructura real del mundo físico. Galileo será un típico representante de la nueva mentalidad que cambia las explicaciones físicas cualitativas de Aristóteles por las formulaciones matemáticas de Arquímedes?.

Con lo anterior se logra una ganancia desde la perspectiva galileana que relaciona los fenómenos con las leyes matemáticas y por tanto numéricas. Es también la insistencia que hace Bacon al afirmar que la naturaleza estaría escrita en caracteres matemáticos para ser interpretados, interpretación que permite transformar la naturaleza. En esta línea argumentativa aparecen autores como Copérnico, Brahe, Kepler y el mismo Galileo hasta llegar a Newton en lo que se conoce como la revolución científica que a su vez implicó una nueva manera de ser del hombre y su relación con el cosmos. Aunque el enfoque está puesto en la matemática como orientadora de la ciencia, lo que se pretende mostrar es que la reflexión tiene un sustento tanto de método como de comprobación experimental en la física y en la naciente química para la época que se está referenciando. Este auge del conocimiento

Aristóteles, Metafísica. Libro I Capítulo I 981a (Madrid: Gredos 1998), 5-10.

Mardones y Ursúa, Filosofía de las ciencias humanas y sociales, 19. 
ya descrito, permitió elaborar una filosofía de la ciencia enunciada en el positivismo de A. Comte y Stuart Mill, que incluso permitió que la sociología fuera contada dentro de las ciencias. La conexión entre lo epistemológico y lo tecnológico determina la cercanía de los conceptos, es decir, la filosofía es el gozne que permite abrir la reflexión entre uno y otro. No es y jamás puede ser una reflexión concéntrica pues no se subsumen los conceptos. Cada concepto tiene su dinámica propia, y la relación y la explicación que se pretende es la que muestra la figura 1. La filosofía es el puente que permite pasar de una orilla a otra y el logos es el empalme o conector entre la episteme y la tecne.

Figura 1: Diagrama de Venn que explica la unión de términos

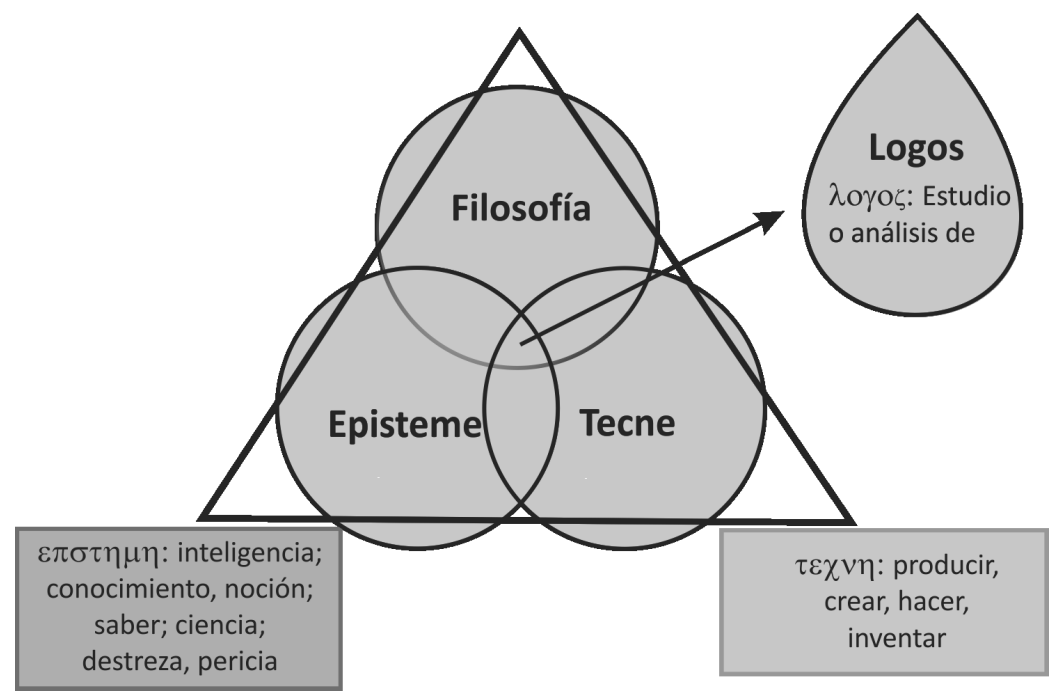

Fuente: Elaboración propia

Aristóteles definió al hombre como «animal racional» $\zeta \omega 0 v \lambda \mathrm{o \gamma ov}$ $\varepsilon \chi O v^{10}$. Logos $(\lambda \mathrm{o} \gamma \mathrm{o} \sigma)$, significó la capacidad de emitir juicios racionales, por lo tanto, tener razón es tener logos. Inmediatamente se genera un hilo conductor entre la razón y el lenguaje, pues los juicios o los enunciados se hicieron expresiones ónticas en las palabras. El pensamiento y lo pensado se expresa por medio del lenguaje hablado y/o escrito. Así las cosas, la concretización del pensamiento se realiza en el discurso con las

$10 \quad$ Aristóteles, Metafisica, 8. 
características que este posee, es decir, válido, coherente y sobre todo, argumentativo, propositivo y crítico. La forma como se mide el ejercicio del pensamiento está en la capacidad argumentativa del discurso, la forma de hilar los enunciados, la lógica de las proposiciones y lo creativo de lo que se dice, como la poesía, por ejemplo. Para este caso de estudio el logos es el punto axial entre episteme, sofos y tecne; en otras palabras, las tres se expresan mediante el logos.

\section{La filosofía de la tecnología ${ }^{11}$}

Debajo de nosotros, emplazado en las playas de una pequeña ensenada, se hallaba el gigante reactor nuclear, todavia en construcción, un enorme bloque marrón rectangular y dos cúpulas blancas. [...] Precisamente en ese instante otra vista me llamo la atención. En línea con el reactory Diablo Rock pero mucho más alejada de la costa, una ballena gris de California repentinamente subió a la superficie, lanzó al aire una columna de vapor de su espiráculo y luego desapareció por debajo de las olas. Un abrumador silencio me dominót2.

¿Es posible hacer filosofía de la tecnología? Claro que sí. En los últimos años se ha escrito mucho al respecto. El origen de la filosofía está dado en la admiración, acción que realiza el individuo humano sobre la realidad cambiante. $\mathrm{Y}$ aquí sí que hay motivos suficientes para admirarse, porque la realidad se ha modificado y en algunos casos, gracias a la técnica. A propósito de esto, para Ortega y Gasset la técnica es: «... la reforma que el hombre impone a la naturaleza en vista de la satisfacción de sus necesidades. [...] reforma en sentido tal que las necesidades queden a ser posible anuladas por dejar de ser problema su satisfacción $\rangle^{13}$. El epígrafe de este apartado orienta el sentido de esta reflexión, es decir, el asombro que causa reconocer que el mundo natural ha cambiado. Seguramente, esto inspiró a Langdon Winner a titular su libro La ballena y el reactor, es uno de los libros sobre filosofía de la tecnología en donde el énfasis es puesto por el autor en el tema político y la razón, tema que ya se encuentra en Platón, al definir la política como el arte de saber

11 El título de este apartado se inspira en la preocupación que se ha generado en el grupo de filósofos que han escrito sobre la particularidad de la reflexión filosófica y los cambios técnicos. Entre ellos están Mitcham, Winner, Ortega y Gasset y Heidegger, entre otros mencionados indirectamente en este trabajo investigativo.

12 Langdon Winner, La ballena y el reactor: una búsqueda de los límites en la era de la alta tecnología (Barcelona: Gedisa, 1987), 188.

13 José Ortega y Gasset, Meditación de la técnica y otros ensayos sobre ciencia y filosofía (Madrid: Alianza, 1982), 28. 
gobernar. Así las cosas, técnica, poesía y política encuentran su relación en el arte.

Carl Mitcham en su prefacio del libro ¿Qué es la filosofía de la tecnología? define la técnica como «conjunto de procedimientos puestos en práctica para obtener un resultado determinado» ${ }^{14}$. No deja de ser una definición algo simple en su planteamiento pero que el autor va argumentando y elaborando a partir de los distintos autores que al respecto se han pronunciado. Al iniciar el libro, Mitcham desarrolla lo que denomina las dos tradiciones de la filosofía de la tecnología, esto es, la filosofía de la tecnología ingenieril y la filosofía de la tecnología de las humanidades. En la primera tradición se destacan los aportes realizados por Ernst Kapp y en la segunda, la referencia es más prolífica pues menciona a Mumford, Ortega y Gasset, Heidegger y Ellul. Estos son algunos de sus aportes:

Ernst Kapp (1808-1896) es quien acuña el término Philosophie der Technik o filosofía de la técnica, y sus primeros intereses investigativos están en torno a la geografía generando una reflexión sobre lo que hoy podríamos denominar una ecología o filosofía del medio ambiente. Kapp es un hegeliano de izquierda y altamente influenciado por Marx, "«emprendió la tarea de formular una filosofía de la tecnología, en la cual los instrumentos y las armas eran entendidos como diferentes tipos de «proyecciones de los órganos» ${ }^{15}$ ".

De la segunda tradición se destaca a Lewis Mumford (1895-1990) cuyo aporte se orienta hacia el Mito de la máquina, (título de una de sus obras) y, sobre todo a explicar los orígenes psicológicos o culturales y las causas materiales y eficientes de la tecnología. Su aporte es muy interesante para este escrito pues realiza una hermenéutica de la acción humana en la simbología del lenguaje que «fue incomparablemente más importante para el ulterior desarrollo humano que el astillar de una montaña de hachas de mano» ${ }^{16}$.

Hasta ahora la elucidación ha girado en torno a la técnica, pero, ¿qué es la tecnología? Se podría afirmar que es el corpus teórico epistemoló-

\footnotetext{
14 Carl Mitcham,¿Qué es la filosofía de la tecnología?(Barcelona: Anthropos. 1989), 12.

15 Mitcham, ¿Qué es la filosofía de la tecnología?, 29.

16 Mitcham, ¿Qué es la filosofía de la tecnología?, 55.
} 
gico que analiza y describe mediante el lenguaje, oral y escrito, la técnica como acción humana. El Diccionario etimológico de Corominas citado por González Quiroz en su libro El porvenir de la razón en la era digital, define tecnología así:

...vocablo que aparece en castellano hacia 1765 (al igual que el nombre de técnico para designar a los especialistas, uso que al final del siglo XIX da lugar a técnica como sustantivo) y se deriva del adjetivo latino technicus que, a su vez, proviene del griego tékhne que significa arte, habilidad, procedimiento, etc. ${ }^{17}$.

Algunos autores se refieren de forma indistinta a la técnica y a la tecnología. Aquí se sugiere que la tecnología es la reflexión filosófico-científica en torno a la técnica. Lo que se está haciendo ahora en este escrito es un tecnologos, o sea un diálogo, una disertación sobre el vasto mundo de la técnica, que no se queda en la mera dialéctica sino que se enmarca y referencia en el lenguaje tanto de ingenieros, como de arquitectos $\mathrm{y}$, como se verá al final, hasta de educadores, reflexión cuyo protagonista principal es el objeto técnico.

\section{a. El objeto técnico}

Los individuos son sistemas de señal-signo. Toda individualidad es intensiva: por consiguiente, es como una cascada, como una esclusa, comunicante, abarcadora, y afirmando en si la diferencia en las intensidades que la constituyen ${ }^{18}$.

Para decir «cosas» los griegos tenían un término adecuado y era prágmata $(\pi \rho \alpha \gamma \mu \alpha \tau \alpha)$, en sí mismo anfibológico, porque podía significar acción, negocio, cosa, hecho, objeto ${ }^{19}$. Lo rescatable de este análisis etimológico es la expresión griega que remite en castellano a pragmático a práctico o al uso de algo; las cosas que tienen un uso puntual se convierten en instrumentos prácticos. «Estos son los objetos técnicos, o sea, las cosas que tienen una finalidad instrumental como herramientas o como utensilios (son útiles al hombre), muchos de ellos se han ido acoplando a las costumbres humanas y no son extraños, por ejemplo la cuchara no es definida como un objeto técnico o instrumento, es a bien un utensilio, es algo familiar, su pragmática, podría decirse es congénita a los humanos. No

\footnotetext{
José Luis Gonzáles, El porvenir de la razón en la era digital (Madrid: Síntesis, 1998), 13.

Gilles Deleuze, Diferencia y Repetición (Buenos Aires: Amorrortu, 2009), 367.

J. M. Pabón, Diccionario Vox Manual Griego (Barcelona: CREMAGRAFIC, 2000), 495.
} 
ocurre lo mismo con un martillo cuyo uso pragmático o práctico es casual y como herramienta es un objeto técnico» ${ }^{20}$. En lo estético, la cultura ha aceptado con facilidad la belleza de la obra de arte, mientras que mantiene cierta reticencia con el objeto técnico, sin desconocer que fenomenológica y existencialmente, no estamos simplemente sumidos en un mundo de cosas pues en medio de ellas están los objetos técnicos.

Para Heidegger las cosas se hacen objetos técnicos o útiles, en su expresión más usual, en su referencia al «para» de su empleo o de su uso. Cuanto mejor se agarre un martillo y se use, más original se vuelve su ser útil, es empleable y, por tanto, como cosa técnica porta la totalidad de referencia en sí misma y se vuelve útil en cuanto encierra una finalidad y está a la mano el habérmelas con la cosa misma. El trato con la cosa la hace útil o no, según sea su referencia con quien hace uso de la cosa, si le es práctica o no para el trabajo que se quiere realizar. Heidegger en su artículo La pregunta por la técnica ${ }^{21}$ dice:

La palabra procede de la lengua griega $\mathrm{T} \varepsilon \chi \mathrm{v} \imath \kappa \mathrm{\kappa}$ quiere decir algo que es de tal modo que pertenece a la $\tau \varepsilon \chi \vee \eta$. En vistas al significado de esta palabra tenemos que prestar atención a dos cosas. En primer lugar $\tau \varepsilon \chi \vee \eta$ no sólo es el nombre para el hacer y el saber hacer del obrero manual sino también para el arte, en el sentido elevado, y para las

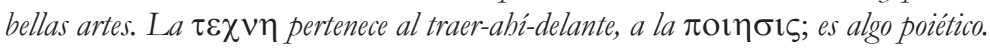

Lo otro que, en vistas a la palabra $\tau \varepsilon \chi \nu \eta$, hay que considerar tiene todavía más peso. La palabra $\tau \varepsilon \chi \vee \eta$, desde muy pronto hasta la época de Platón, va de consuno con la palabra $\varepsilon \pi \imath \sigma \tau \eta \mu \eta$. Ambas palabras son nombres para el conocer en el sentido más amplio. Lo que ellas mientan es un entender en algo, ser entendido en algo. En el conocer se hace patente algo. [...] La $\tau \varepsilon \chi \nu \eta$ es un modo del $\alpha \lambda \eta \theta \varepsilon v \varepsilon i v$. Saca de lo oculto algo que no se produce a si mismo y todavía no se halla abi delante, y por ello puede aparecer y acaecer de este modo o de este otro. El que construye una casa o un barco o forja una copa sacrificial hace salir de lo oculto lo-que-hay-que-traer-abi-delante, y lo hace según las perspectivas de los cuatro modos del ocasionar. ${ }^{22}$

Uno de los grandes desconocidos de la filosofía de la técnica es el filósofo Gilbert Simondon, autor de El modo de existencia de los objetos

20 Ha sido una preocupación en la indagación de quien escribe estas líneas, realizar un estudio juicioso de lo que es el objeto técnico y su impacto en el espacio educativo. Al respecto véase: José Alberto Rivera P., "Una hermenéutica de la educación a distancia desde una filosofía de la técnica", Revista de investigaciones UNAD, vol. $9 \mathrm{~N}^{\circ}$ 3 (2010), 7.

21 Martín Heidegger, Conferencia pronunciada en la Academia Bávara de las Bellas Artes, en Vörtrageund Aufsätze, 1953.

22 Martín Heidegger, Conferencias y artículos. (Barcelona: Ediciones del Serbal, 1994), 24. 
técnicos. A él se le ha denominado el abogado del objeto técnico y el pedagogo de las máquinas; es además, un crítico de aquellos que satanizan la técnica y la ponen en otro nivel. Este filósofo francés hace la reflexión en términos propios del fenómeno técnico, es decir, llamando cada cosa con su nombre desde el ámbito de la electricidad, la electrónica o la industria en general. Se trata de una reflexión filosófica con términos ingenieriles, con un lenguaje propio.

Más arriba se ha dicho que el logos permite transitar entre la ciencia de las ciencias (epistemología) y la tecnología, lo que equivale a decir que el lenguaje permite mostrar la cercanía y que el protagonista de esta dialéctica es necesariamente el objeto técnico. ¿Por qué adquiere mayor significado protagónico una obra de arte que un objeto técnico? ¿Acaso no se puede decir que un motor, sea cual sea, es también bello?

Por esto el descubrimiento de la belleza de los objetos técnicos no puede ser abandonada unicamente a la percepción: hace falta que la función del objeto sea comprendida y pensada; dicho de otro modo, hace falta una educación técnica para que la belleza de los objetos técnicos pueda aparecer como inserción de los esquemas técnicos en un universos, en los puntos-claves de este universo ${ }^{23}$.

Simondon se mueve en una explicación triangular: religión, tecnicidad y estética ${ }^{24}$; estas también llamadas fases, que en su desplazamiento o desfase se originan la una de la otra y a su vez en cada una se da un desdoblamiento en un modo teórico y un modo práctico. En el campo de lo estético, Gadamer ha estudiado el carácter óntico de la obra de arte y ha dicho que en cuanto repraesentatio (representación), el cuadro es manifestación artística, es mimesis de imagen ${ }^{25}$. De alguna manera esto explica por qué en el mundo de las cosas una obra de arte, cualquiera que sea su manifestación, adquiere derecho de existencia y de importancia ontológica al ser reconocida por la cultura, mientras que un martillo, un hacha, un computador, remite inmediatamente a su función unilateral, utilitarista y de maniobra externa. ¿Cómo gana espacio ontológico el objeto técnico?

Gilbert Simondon, El modo de existencia de los objetos técnicos (Buenos Aires: Prometeo, 2007), 203.

Gilbert Simondon "El modo de existencia de los objetos técnicos", 206.

Gadamer en La actualidad de lo bello y en Verdad y método I, realiza un estudio fenomenológico de la obra de arte en el sentido cognitivo de la imitación. Son dos los elementos a resaltar en esta concepción: el carácter lúdico de la obra de arte y su posibilidad de ser conocida al jugar imitando, lo cual llama la atención 


\section{b. El estatuto ontológico del objeto técnico}

Con Simondon el objeto técnico adquiere una dimensión ontológica, algo compleja de comprender; pues le denomina individuo. El objeto técnico se hace individuo y la condición previa de la individuación se da como respuesta a una necesidad, es decir, suple una carencia real. Fenomenológicamente es claro, que un objeto es una cosa en medio de las cosas; mientras que el individuo adquiere una representación óntica distinta, dada por su autodeterminación que le hace sujeto y por tanto distinto del objeto-cosa. A pesar de lo complejo que en un primer momento pueda significar:

El principio de individualización del objeto técnico a través de la causalidad recurrente dentro del medio asociado permite [...] saber si es preciso tratarlos como individuo técnico o como colección organizada de individuos. Diremos que hay individuo técnico cuando el medio asociado existe como condición sine qua non de funcionamiento, mientras que hay conjunto en el caso contrario ${ }^{26}$.

El objeto técnico también funciona como mediador de inteligencia colectiva tal y como lo plantea Pierre Levy en su libro ¿Qué es lo virtual? Porque es testigo de cambios y de progresos, el objeto está entre las manos de unos y otros. Es atravesado por las distintas operaciones que deliberadamente el sujeto hace de él y lo pone en contacto con lo real. «Nuestra subjetividad se abre al juego de los objetos comunes que tejen con un mismo gesto simétrico y complicado la inteligencia individual y la inteligencia colectiva, como el anverso y el reverso del mismo tejido» ${ }^{27}$. Valga aquí la oportunidad de plantear una cercanía entre las mediaciones pedagógicas de la educación apoyada en las TIC (Tecnologías de la Información y la Comunicación) y el sujeto que aprende.

\section{c. La evolución o concretización del objeto técnico.}

De lo que se trata en este apartado, es de explicar el proceso de concretización, de casi evolución que alcanza el objeto técnico; de la tecnicidad como generadora de objetos técnicos

desde el horizonte educativo para hacer del aprendizaje un espacio lúdico de conocimiento. Más adelante en

la última parte se mencionará el ambiente virtual de aprendizaje como espacio dinámico de conocimiento.

26 Gilbert Simondon, El modo de existencia de los objetos técnicos, 81.

27 Pierre Lévy, ¿Qué es lo virtual?(Barcelona: Paidós, 1999),119. 
La invención que es creación del individuo, supone en el inventor el conocimiento intuitivo de la tecnicidad de los elementos; la invención se cumple en ese nivel intermedio entre lo concreto y lo abstracto[...]. En la reflexión acerca de las consecuencias del desarrollo técnico en relación con la evolución de las sociedades humanas, lo que hay que tener en cuenta, antes que nada, es el proceso de individualización de los objetos técnicos; la individualidad humana se encuentra cada vez más separada de la función técnica por la construcción de individuos técnicos... ${ }^{28}$.

Afortunadamente la historia de la humanidad se ha visto beneficiada con el proceso de concretización del objeto técnico. Esto, de entrada permitirá reafirmar el carácter apologético que Simondon esgrime ante los ataques satanizadores y denigrantes que se hacen sobre la técnica y los avances tecnológicos. El objeto técnico, entonces, después de dejar una fase artesanal alcanza su concretización en el lugar intermedio entre el objeto natural y la representación científica, pues sus acciones se ejercen sobre las leyes de la ciencia. «Sólo son inventados, para hablar con propiedad, los objetos técnicos que precisan un medio asociado para ser viables.... ${ }^{29}$. Un computador es posible, hoy por hoy, porque su medio asociado le permite existir, porque hay un requerimiento del medio, porque es una necesidad histórica en cuanto que es una evolución de la humanidad. Lo particular y propio del objeto le permite ser tal por el proceso de concretización, el cual se proyecta hacia el futuro.

Independientemente de la reflexión filosófica que se haga sobre el objeto técnico, este existe y no necesita permiso alguno para ser tal. La lógica de la programación, los algoritmos, los microprocesadores, la telefonía celular, el iPhone y tantas concretizaciones que se han dado del objeto técnico permiten reafirmar lo positivo de la inventiva humana. La invención es una operación mental que pone en la realidad una complejidad que supera la saturación de la máquina para que le permita al hombre ser más, en lo que se refiere al carácter óntico. «...esto es, el objeto técnico en la medida en que ha sido inventado, pensado y querido, asumido por un sujeto humano, se convierte en el soporte y el símbolo de esta relación que querríamos denominar transindividuali ${ }^{30}$. Lo anterior le permite, incluso a Simondon llegar a afirmar que en el objeto inventado hay algo de la naturaleza de su inventor hay naturaleza humana en el ser técnico. Así, la

\footnotetext{
Gilbert Simondon, El modo de existencia de los objetos técnicos, 100.

Simondon, El modo de existencia de los objetos técnicos,78.

Gilbert Simondon, El modo de existencia de los objetos técnicos, 263.
} 
criatura dependería de su creador y la técnica del técnico. Esta afirmación que tranquilizaría la inquietud que tienen algunos sobre el manejo e independencia del mundo técnico; pues «... los objetos aparecen en un cierto momento, pero la tecnicidad los precede y los supera; los objetos técnicos resultan de una objetivación de la tecnicidad; son producidos por ella, pero la tecnicidad no se agota en los objetos y no está totalmente contenida en ellos» ${ }^{31}$. Así como la ousía (substancia) aristotélica, permite dar sostén a la realidad de las cosas, la tecnicidad permite justificar la aparición del objeto técnico, la finalidad o la teleología de la técnica se da en el proceso de concretización del objeto, proceso alcanzado por la sobresaturación del mismo.

Aunque el proceso de aceleración ha sido incalculable y sorprendente y todo lo que queda por avanzar y descubrir es demasiado amplio. Por ejemplo, el internet actualmente ha hecho más compleja la autopista electrónica. Simondon lo vislumbró de alguna manera al referirse al tema de la reticularidad.

Por ello, en la medida en que una tecnología politécnica reemplace (sic) a las técnicas separadas, las realidades técnicas mismas, en su objetividad realizada, adoptan la estructura de red, están en relación unas con otras, [...] pero los conjuntos técnicos son verdaderas redes vinculadas concretamente con el mundo natural [...] pero no se cambia una red, no se construye una red por sí mismo: sólo se puede conectar con la red, adaptarse a ella, participar de ella; la red domina y ciñe la acción del ser individual, domina incluso cada conjunto técnico ${ }^{32}$.

Sin forzar a nadie a decir algo distinto, la interpretación actual de este apartado bien podría hacerse desde la internet. La cultura humana ha sido modificada por esta tecnología, la relación del hombre con el mundo y consigo mismo han cambiado, la cultura ha sido impregnada por este ambiente virtual.

\section{Tecnología educativa ${ }^{33}$}

La educación es, posiblemente, la rama más importante para la técnica científica, puesto que influye enormemente en toda nuestra existencia. No debemos seguir permitiendo que

\footnotetext{
Gilbert Simondon, El modo de existencia de los objetos técnicos, 180.

Gilbert Simondon, El modo de existencia de los objetos técnicos, 236-237.

33 La tecnología educativa es actualmente un tema de gran interés, muestra de lo cual es la amplia bibliografía y la preocupación por dar las orientaciones respectivas, sobre todo cuando implican un reto educativo. En este escrito se ha tomado a Cabero con su libro Tecnología educativa. (Madrid: McGraw-Hill, 2007).
} 
las exigencias de una situación práctica impidan los inmensos adelantos que están a nuestro alcance. $[\ldots]$ Los niños se entretienen durante horas enteras con juguetes mecánicos, lápices de colores, papel y tijeras, artefactos que meten ruido, rompecabezas...; en una palabra, con casi todo lo que introduce cambios significativos en el ambiente y está razonablemente libre de propiedades ingratas o aversivas ${ }^{34}$.

Las coordenadas ya están puestas y bien delineadas, una es la técnica y otra es la episteme o ciencia, en ese plano ¿dónde se ubica la educación? Es necesario decir que la educación como proceso humano jamás ha estado apartada de los impactos positivos de los avances técnicos, por el contrario se ha enriquecido con los mismos. Es posible pensar que la pizarra, la tiza, el lápiz, el cuaderno y el texto son objetos técnicos que han facilitado la enseñanza y el aprendizaje a lo largo de la historia humana.

El computador-objeto técnico concretizado, tanto en software, como en hardware- ha facilitado procesos educativos ${ }^{35}$, posibles por los avances en los procesadores de texto, de gráficos, de tablas, etc. En definitiva,

... la realidad que se nos construye en las pantallas no solo influye sobre nuestra conducta, sino también sobre nuestra percepción del mundo haciéndonos vivir frecuentemente en una realidad que es en cierto modo virtual, pero que desde los propios medios puede presentarse como la realidad-real ${ }^{36}$.

Acorde con la cita anterior, hoy se habla de interfaces inteligentes, es decir, de ambientes que poseen todas las características necesarias para simular y crear dimensiones y coordenadas virtuales que facilitan un aprendizaje real en cuanto la información suministrada sea auténtica y veraz. Algo para destacar en este orden de ideas es el aporte de Nicholas Negroponte respecto a la enseñanza de los niños a través del juego, cuando señala:

Los chicos que hoy usan LEGO/Logo aprenderán principios físicos y lógicos que ustedy yo aprendimos en la universidad. La evidencia anecdótica y los resultados de pruebas minuciosas revelan que este enfoque constructivista es un medio de extraordinaria riqueza para aprender a través de una amplia variedad de estilos cognitivos y de comportamiento. De hecho, muchos niños que se suponian que eran incapaces de aprender, mejoraron en el entorno constructivista ${ }^{37}$.

\footnotetext{
34 Burrhus F. Skinner, Tecnología de la enseñanza (Barcelona: Labor, 1970), 34.

35 Para ampliar este tema se puede revisar el capítulo 11 del libro de Julio Cabero, Tecnología educativa (Madrid: McGraw-Hill, 2007), 173-193.

36 José López, José Luján y Eduardo García, Filosofía de la tecnología (Madrid: OEI, 2001), 89.

37 Nicholas Negroponte, El mundo digital. Un futuro que ya ha llegado (Barcelona: Ediciones B, S. A, 2000), 237.
} 
El juego tiene unas características importantes que se aprovechan hoy en la educación apoyada en los medios técnicos. Entre estas tenemos su carácter dinámico, autónomo y colaborativo. Aprender jugando no es solamente un imperativo para la educación infantil, ya que es válido también en los distintos momentos de la formación humana y con mayor razón cuando se dispone de las herramientas para construir espacios dinámicos gracias a la multimedia, los simuladores y los laboratorios remotos; la lista continuará, gracias a lo cual cada vez se aprenderá de forma distinta aunque prevalezcan las mismas características del juego mencionadas antes.

Hoy la pedagogía está mediada por la tecnología o la didáctica, las cuales se apoyan en los medios técnicos ${ }^{38}$, de tal manera que su realidad se impone, utilizando las tan mentadas TIC como soporte y facilitadoras de los procesos de enseñanza y de aprendizaje. Lo anterior no significa que de manera exclusiva, estemos en un momento histórico privilegiado para la educación, pues en otros momentos de la historia los avances de tipo técnico o los nuevos descubrimientos han permitido un progreso en la educación, baste por ahora mencionar el invento de la imprenta y por que no, del lápiz y del papel.

La escritura ha perdido su continente, en palabras de Lévy se habla de la desterritorialización del texto ${ }^{39}$. Esto ha permitido que la información sea fácilmente accesible. Así las cosas, la Web ha revolucionado el mundo de la educación, a tal punto que hoy está bien ${ }^{40}$ hablar de ambiente virtual de aprendizaje, y todo esto simplemente porque:

La virtualización, lejos de aniquilar el texto, parece hacerlo coincidir con su esencia desvelada de repente. Es como si la virtualización contemporánea cumpliese el destino del texto, como si saliésemos de una cierta prehistoria y la aventura del texto se acabara de iniciar, como si, en definitiva, acabáramos de inventar la escritura ${ }^{41}$.

38 Elena Barberày Antoni Badia. Educar con aulas virtuales. Orientaciones para la innovación en el proceso de enseñanza y aprendizaje (Madrid: Machado libros, 2004), 4. Para estos autores, tecnología "debe ser un medio para alcanzar fines educativos".

39 Lévy, ¿Qué es lo virtual?, 45.

40 Sobre este aspecto vale la pena mencionar que algunos autores manifiestan ciertas disonancias sobre el tema del hipertexto, Kerckhove, previene sobre el poder que ejerce el texto sobre el lector ya que "puede controlar al lector por el puro poder, velocidad y complejidad de sus operaciones". Derrick de Kerckhove, Inteligencias en conexión (Barcelona: Gedisa, 1999), 127.

41 Lévy, ¿Qué es lo virtual?, 47. 
En 1958 B. F. Skinner escribe un artículo titulado "Teaching Machines"; de manera más directa empezó a escribir sobre la máquina de enseñar en un contexto histórico enmarcado en el lanzamiento del primer Sputnik, el radio y el televisor de tubos, el teléfono superando al telégrafo y otros avances de la revolución industrial. José Castro, autor del prólogo del libro de Skinner escribe que en dicho contexto surgió la expresión: «Tecnifiquemos la educación», con lo cual mejoraría el sistema educativo de aquel entonces. Así mismo se aplicó al proceso de aprendizaje, dando origen a las máquinas de enseñar, de las cuales, las primeras enseñaban a hacer cálculos numéricos similares a la de Babbage y otras enseñaban a deletrear a estudiantes de tercer grado. Con todo ello, debemos aceptar que la educación ha llegado a esta fase algo tarde,

...quizá por no haber sido bien comprendida su tarea. Sin embargo, gracias al invento de la televisión, las llamadas ayudas audiovisuales están siendo reconsideradas. Los proyectores de películas cinematográficas y diapositivas, los aparatos de television, los tocadiscos y los magnetófonos se están introduciendo cada vez más en las escuelas y en los colegios de América. Los recursos audiovisuales complementan y hasta pueden suplir a las lecturas, demostraciones y obras de texto escolares ${ }^{42}$.

La presentación de Skinner en este sentido es válida siempre y cuando se reconozcan sus aportes desde el objeto técnico concretizado en la máquina de enseñar. Sin embargo, es necesario hacer énfasis en algunos matices respecto del papel fundamental que actualmente tienen el estudiante y el docente, muy distinto de la concepción que tenía el psicólogo del aprendizaje en los años sesenta del siglo pasado. Capacho llama la atención sobre este particular al criticar ciertos entornos de aprendizaje:

La teoría del condicionamiento de Skinner, en la secuencia $E / R / Z$, convierte en el aprendizaje en una relación de entrada-salida. Luego, en función de esta relación, los procesos de formación son soportados por ambientes de aprendizaje virtual basados en TIC que funcionan a nivel de entradas y salidas, similar al funcionamiento de los componentes del hardware y el software en las TIC, que funcionan a nivel de entradas-salidas; y en la mayoría de los casos, para usuarios no técnicos, la funcionalidad interna de la componente base funciona como una "caja negra", similar al concepto de caja negra de la teoría conductista ${ }^{43}$.

42 Burrhus Frederic Skinner, Tecnología de la enseñanza, 43.

43 José Capacho, Evaluación del aprendizaje en espacios virtuales-TIC (Bogotá: ECOE, 2011), 79. 
Los ambientes virtuales de aprendizaje son la mejor concretización que ha alcanzado la educación gracias a la técnica. La virtualidad ${ }^{44}$ es un medio favorable en el fortalecimiento de la pedagogía y brinda herramientas que permiten avanzar en la didáctica, empero, es necesario atender a los llamados de atención para no caer en teorías otrora ya superadas. Por el contrario se trata de revalidar y rescatar las características propias del aprendizaje que se da en tales espacios, donde el estudiante es el protagonista del aprendizaje y asume de manera autónoma dicho proceso con las orientaciones del docente-tutor. Hoy las máquinas de enseñar hacen uso de los recursos multimedia, que le permiten al estudiante que ingresa al ambiente virtual estar recibiendo información sensorial casi que a través de los cinco sentidos, es una dinámica totalmente distinta a la que se da en el ambiente tradicional del aula donde el maestro instruye desde la cátedra.

La tecnología y la educación afortunadamente han encontrado su relación y sobre el tema se ha escrito y hay un gran interés para avanzar sobre todo reconociendo las bondades que aporta lo tecnológico a lo educati$\mathrm{vo}^{45}$. Esto ha permitido hacer de la educación algo interactiva y disponer de espacios distintos a los convencionales para educar, el e-learning ${ }^{46} \mathrm{O}$ aprendizaje electrónico se ha insertado en la dinámica del mundo globalizado y ha permitido abrir las fronteras del conocimiento. Desde luego que toda la educación en su naturaleza ha sido afectada positivamente en estos cambios, a pesar de las dificultades que pueda implicar educar desde el aula tradicional porque los recursos económicos ${ }^{47}$ sean escasos, o los niveles económicos no lo permitan. Los estudiantes viven en el mundo de lo digital y a pesar de las circunstancias, sus vidas están inmersas en una realidad a la que hay que responder con los mínimos posibles, pero sin esconderles y sin escatimar los esfuerzos que se requieran para brindar la educación con recursos de tipo tecnológico, por algo al comienzo de este apartado se decía que el cuaderno, el texto y el lápiz son objetos técnicos.

44 Para Cardona «... la Educación virtual sea la que mejor combina el trabajo con el estudio, la que tiene mayores posibilidades de resolver este problema por cuanto puede considerar las experiencias vitales como parte de la evaluación, de acuerdo con el reconocimiento o rechazo que la misma sociedad haga de sus éxitos y fracasos respectivamente...» Guillermo Cardona. "Tendencias educativas para el siglo XXI, educación virtual, On Line, y @learning. Elementos para la discusión”, Edutec No. 15 (2002), 10.

45 Julio Cabero, Tecnología educativa. (Madrid: McGraw-Hill, 2007), 34.

46 Elena Barberà, Aprender e-learning. (Barcelona: Paidós, 2008), 13.

47 Manuel Castells, La era de la información, vols. I-III. (Madrid: Alianza, 2000), 407. 
La tecnología educativa hoy no permanece hermética, por el contrario asume algunos desafíos los cuales le permiten dinamizar y reconstruir desde el currículo hasta la generación de los nuevos medios que entran a apoyar la tarea del docente y permite:

...transformar radicalmente sus modelos didácticos en todas las áreas del currículo; a medida que el docente se las apropia, cambia su papel tradicional y también se derrumban los limites tradicionalmente impuestos al currículo, permitiendo que las diversas disciplinas se integren e interactúen en la realización de tareas y proyectos ${ }^{48}$.

Se habla entonces de la efectividad del diseño instruccional y de la ecología formativa, creando así una estela todavía más compleja a la cual es necesario seguirle el rastro. Autores como Barberà, Cabero, Sangrà; entre otros de lengua española han enriquecido la reflexión sobre este aspecto. Vale la pena reiterar que la educación está ubicada en un contexto histórico favorable y despierta un interrogante de sana preocupación por el futuro, sobre todo porque el docente en sus nuevos roles debe repensar su papel y su deber hacer desde los ámbitos ya descritos ${ }^{49}$. Es importante tener puesta la mirada en el proceso de concretización y de sobresaturación del objeto técnico y sus nuevos requerimientos y apoyos para el inmenso horizonte educativo.

\section{CONCLUSIÓN}

En suma, los avances técnicos han sido vastos, la mirada retrospectiva no abarca todo lo que se debería visualizar. Pero el encuentro de la educación y la técnica han sido de beneficio, riqueza y complementariedad. La reflexión termina en un punto álgido puesto que se sugiere dar un nuevo paso y avanzar implica analizar y estudiar los pendientes en cuanto a las repercusiones con la didáctica y hasta con las políticas educativas. La educación no es un simple catalizador de los procesos que implica la relación entre tecnología y el conocimiento científico, debe ser más bien el vehículo que de visibilidad a

48 Manuel Cebrián, Tecnologías de la información y comunicación para la formación de docente (Madrid: Ediciones Pirámide, 2009), 35.

49 Sobre este aspecto se puede ampliar lo que Sangrà afirma lo siguiente: «Con el objetivo de hacer llegar la educación a todo aquel que la necesita, aparecieron las prácticas de educación a distancia. Estas prácticas han exigido siempre la existencia de un elemento mediador entre el docente y el discente. Generalmente, este mediador ha sido una tecnología, que ha ido variando en cada momento". Albert Sangrà, "Enseñar y aprender en la virtualidad". Revista EDUCAR, No. 28 (2001), 122. 
los avances, sobre todo cuando estos permiten dinamizar nuevos procesos de aprendizaje. La educación debe adaptarse tanto a los avances técnicos, como a las nuevas exigencias didácticas y pedagógicas, que retan en la implementación de nuevas orientaciones desde el campo educativo. Es perentorio $^{50}$ que el proceso educativo sea eficaz, efectivo para el hombre ubicado en este contexto cambiante, suficientemente ya descrito.

El centro del escrito giró en torno al objeto técnico, la tarea continúa en la revisión de otros objetos técnicos que se ubican en el ciberespacio y que responden también a la educación. No se trata del artefacto hermético e inerte, el rescate es hacia la dinámica que confiere y desarrolla la nueva didáctica en la enseñanza desde los ambientes virtuales de aprendizaje. Un nuevo reto se vislumbra desde ya, se trata de continuar investigando sobre esta nueva realidad de la educación apoyada en la técnica, pero colocando la mirada en el sujeto que se educa. Se quiere indagar en la subjetividad del cyborg ${ }^{51}$, el nuevo individuo que habita el ciberespacio. La tarea en esta dimensión continúa.

\section{REFERENCIAS}

Adell, Jordi. "Tendencias en educación en la sociedad de las tecnologías de la información”. Revista Electrónica de Tecnología Educativa, vol. 5 No 7 (1997): 14-25.

Adell, Jordi; Castellet, José Miguel y Gumbau, José Pascual. Selección de un entorno virtual de enseñanza/ aprendizaje de código fuente abierto para la Universitat Jaime I. Castellón, España: Centre d'Educació i Noves Tecnologies (CENT) de la Universitat Jaume I, 2011. http://cent.uji.es/ doc/eveauji es.pdf

Aguilar, Teresa. Ontología cyborg. Barcelona: Gedisa, 2008.

Aristóteles. Metafísica, Madrid: Gredos, 1998.

Barberà, Elena y Badía, Antoni. Educar con aulas virtuales. Orientaciones para la innovación en el proceso de enseñanza y aprendizaje. Madrid: Machado libros, 2004.

Barberà, Elena y Badia, Antoni. "El uso educativo de las aulas virtuales emergentes en la educación superior”. Revista de Universidad y Sociedad del Conocimiento, vol. 2 No. 2. (2005): 1-12.

50 Hoy se habla de aprendizaje en red en estos términos: «La gestión de ese conocimiento a través de los nuevos procesos de comunicación con el uso de redes tecnológicas, los nuevos canales de información y los contenidos digitales, son actualmente la materia prima o sustancia base de muchos procesos de formación a distancia, con lo que se constituye en el recurso estratégico del desarrollo actual y se coloca en el centro de los procesos de transformación social». Carles Dorado. "El trabajo en red como fuente de aprendizaje: posibilidades y límites para la creación de conocimiento. Una visión crítica”. Revista EDUCAR, No. 37 (2006), 14.

51 El ensayo escrito por Teresa Aguilar titulado Ontología cyborg da luces sobre este punto tan particular y además, génesis de una nueva indagación. 
Barberà, Elena. Aprender e-learning. Barcelona: Paidós, 2008.

Bedoya, José. Pedagogía ¿Enseñar a pensar? Reflexión filosófica sobre el proceso de enseñar. Bogotá: ECOE, 2008.

Bunge, Mario. Epistemología. México: Siglo XXI Editores, 1980.

Cabero Almenara, Julio; Salinas, Jesús; Aguaded G., José Ignacio. Tecnologías para la educación: diseño, producción y evaluación de medios para la formación docente. Madrid: Alianza Editorial, 2004.

Cabero Almenara, Julio. Tecnología educativa. Madrid: McGraw-Hill, 2007.

Cabero Almenara, Julio. "Bases pedagógicas del e-learning". Revista de Universidad y Sociedad del Conocimiento, vol. 3, No 1 (2006).

Capacho, José. Evaluación del aprendizaje en espacios virtuales-TIC. Bogotá: ECOE, 2011.

Cardona, Guillermo. “Tendencias educativas para el siglo XXI, educación virtual, On Line, y @learning. Elementos para la discusión”, Edutec (2002) 1-25 http://edutec.rediris.es/Revelec2/revelec15/cardona.pdf

Castells, Manuel. La era de la información, vols. I-III. Madrid: Alianza, 2000.

Castillo, Santiago y García Aretio, Lorenzo. El tutor y la tutoría en el modelo UNED. Madrid: Dialnet, 1996.

Cebrián, Manuel. Tecnologias de la información y comunicación para la formación de docentes. Madrid: Ediciones Pirámide, 2009.

Crook, Charles. Ordenadores y aprendizaje colaborativo. Ministerio de Educación y cultura. Madrid: Moratta S.L., 1998.

De Camilloni, Alicia. Los obstáculos epistemológicos en la enseñanza. Madrid: Gedisa, 2001.

Duart, Josep. Aprender en la virtualidad. Barcelona: Gedisa, 2000.

Deleuze, Gilles. Diferencia y repetición. Buenos Aires: Amorrortu, 2009.

Domínguez, Guillermo; Torres, Luisa y López, Eloy. Aprendizajes con Wikis. Usos didácticos y casos prácticos. Bogotá: Ediciones de la U, 2010.

Dorado, Carles. "El trabajo en red como fuente de aprendizaje: posibilidades y límites para la creación de conocimiento. Una visión crítica". Revista EDUCAR, N 37. (2006): 11-24. http://ddd.uab.es/pub/educar/0211819Xn37p11.pdf

Duart, Josep. Aprender en la virtualidad. Barcelona: Gedisa, 2000.

Ellul, Jacques. La edad de la técnica. Barcelona: Octaedro, 2003.

Gadamer, Hans G. La actualidad de lo bello. Barcelona: Paidós, 1991.

Gadamer, Hans G. Verdad y método I. Salamanca: Sígueme, 1999.

García Aretio, Lorenzo. Pasado y presente de la acción tutorial en la UNED. Madrid: UNED, 1999.

García Aretio, Lorenzo. La educación a distancia. De la teoría a la práctica. Barcelona: Ariel Educación, 2002. 
García Aretio, Lorenzo; Ruiz, Marta, y Domínguez, Daniel. De la educación a distancia a la educación virtual. Barcelona: Ariel, 2007.

González Quirós, José. El porvenir de la razón en la era digital. Madrid: Síntesis, 1998.

Gramsci, Antonio. La alternativa pedagógica, México: Fontamara, 2007.

Heidegger, Martín. Conferencias y artículos. Barcelona: Ediciones del Serbal, 1994.

Hoyos, Guillermo. Filosofía de la educación, Madrid: Trotta, 2008.

Humbert, Juan. Mitología griega y romana. Barcelona: Editorial Gustavo Gili S. A., 1997.

Jaeger, Werner. Paideia: los ideales de la cultura griega. México: Fondo de Cultura Económica, 1948.

Kerckhove, Derrick de. Inteligencias en conexión. Barcelona: Gedisa, 1999.

Kuhn, Thomas. La estructura de las revoluciones cientificas. México: Fondo de Cultura Económica, 2004.

Lévy, Pierre. ¿Qué es lo virtual? Barcelona: Paidós, 1999.

Lévy, Pierre. Cibercultura. La cultura de la sociedad digital. Barcelona: Anthropos, 2007.

Litwin, Edith y Cerrotta, Cecilia. Tecnología en las aulas. Las nuevas tecnologías en las prácticas de enseñanza. Casos para el análisis. Buenos Aires: Amorrortu Editores, 2005.

López, José; Luján, José y García, Eduardo. Filosofía de la tecnología. Madrid: Organización de Estados Iberoamericanos OEI, 2001.

Mardones, José y Ursúa, Nicolás. Filosofía de las ciencias humanas y sociales. Barcelona: Anthropos, 1983.

McLuhan, Marshall y Powers, Bruce. La aldea global. Barcelona: Gedisa, 2005.

Mitcham, Carl. ¿Qué es la filosofía de la tecnología? Barcelona: Anthropos, 1989.

Negroponte, Nicholas. El mundo digital. Un futuro que ya ha llegado. Barcelona: Ediciones B. S. A., 2000.

Ortega y Gasset, José. Meditación de la técnica y otros ensayos sobre ciencia y filosofía. Madrid: Alianza, 1982.

Pabón, José María. Diccionario Vox Manual Griego Barcelona: CREMAGRAFIC, 2000.

Parra, Jaime. Quebrantos de la mente. Bogotá: Gobernación de Cundinamarca - PUJ, 2004.

Rivera Piragauta, José Alberto. "Una hermenéutica de la educación a distancia desde una filosofía de la técnica", Revista de investigaciones UNAD, vol. 9 No 3 (2010): 7-23.

Rosales, Amán. Filosofía de la tecnología. Bogotá: San Pablo, 2009.

Sangrà, Albert. "Enseñar y aprender en la virtualidad". Revista EDUCAR, vol. 28 (2001): 117-131. http://ddd.uab.es/pub/educar/0211819Xn28p117.pdf

Silva, Marco. Educación interactiva. Enseñanza y aprendiraje presencialy on line. Barcelona: Gedisa, 2008. 
Simondon, Gilbert. El modo de existencia de los objetos técnicos. Buenos Aires: Prometeo, 2007.

Skinner, Burrhus Frederic. Tecnología de la enseñanza. Barcelona: Labor, 1970.

Winner, Langdon. La ballena y el reactor: una búsqueda de los límites en la era de la alta tecnología. Barcelona: Gedisa, 1987.

Zubiría de, Miguel. Enfoques pedagógicos y didácticas contemporáneas. Bogotá: Fundación Internacional de Pedagogía Conceptual, FIPC, 2007.

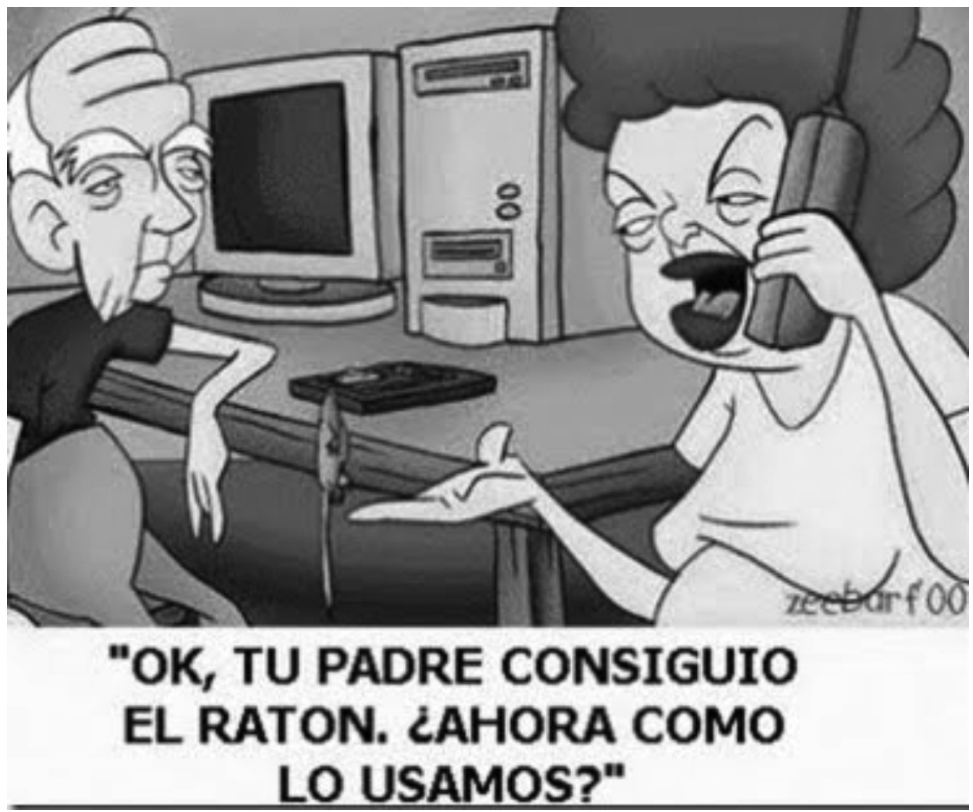

Fuente: http://2.bp.blogspot.com/_MRwsv_CHt1g/Swyp4f1iBuI/AAAAAAAAABk/MA k8wAxpj0g/s400/padreRaton.jpg 


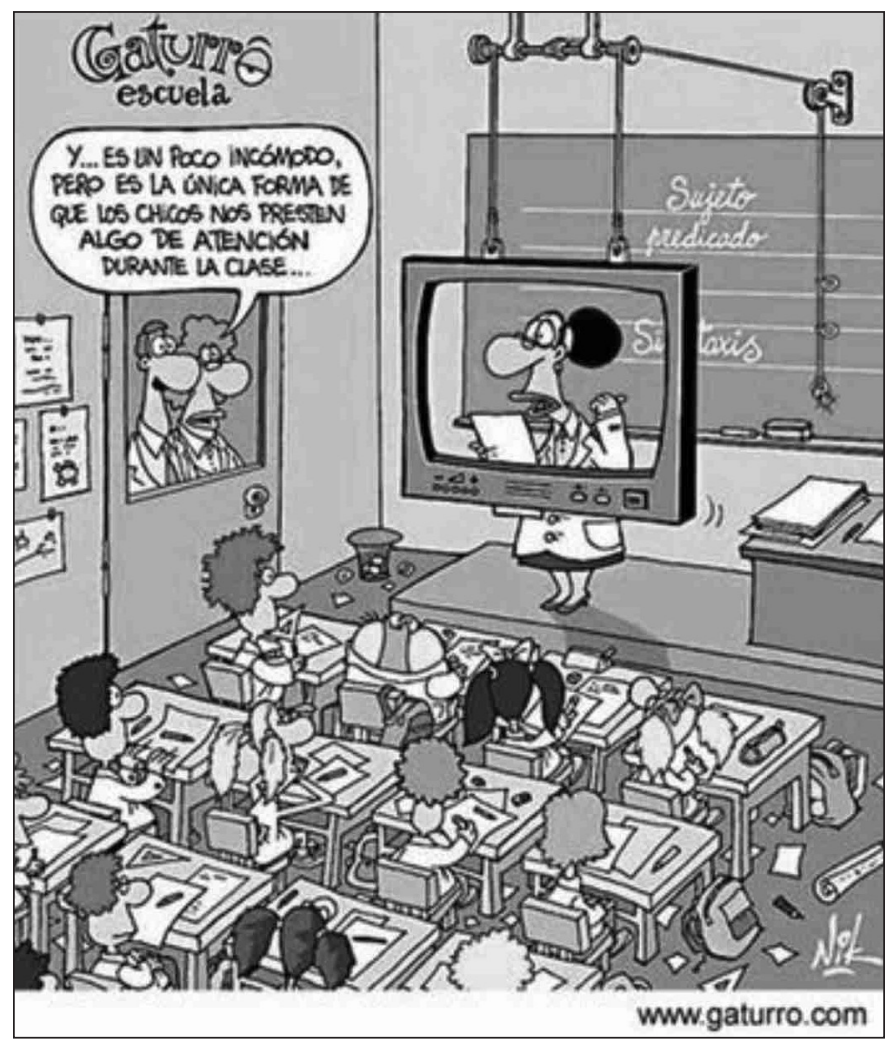

Fuente: http://2.bp.blogspot.com/_yLZO6VZ435c/TQKSzlvHHbI/AAAAAAA AAAU/JXK5m48akHQ/s1600/humor20tics2020091.jpg

Rivera Piragauta, José Alberto. "La educación, entre la ciencia y la técnica". Revista Historia de la Educación Latinoamericana. Vol. 14 No. 19, (2012): 